\title{
ATF4 Contributes to Ovulation via Regulating COX2/PGE2 Expression: A Potential Role of ATF4 in PCOS
}

\author{
Fangfang $\mathrm{Di}^{1,2+}$, Jiansheng Liu ${ }^{1,2 \dagger}$, Shang $\mathrm{Li}^{1,2 \dagger}$, Guangxin Yao ${ }^{1,2}$, Yan Hong ${ }^{1,2}$, \\ Zi-Jiang Chen ${ }^{1,2,3}$, Weiping $\mathrm{Li}^{1,2}$ and Yanzhi Du ${ }^{1,2 *}$ \\ ${ }^{1}$ Center for Reproductive Medicine, School of Medicine, Ren Ji Hospital, Shanghai Jiao Tong University, Shanghai, China, \\ ${ }^{2}$ Shanghai Key Laboratory for Assisted Reproduction and Reproductive Genetics, Shanghai, China, ${ }^{3}$ The Key Laboratory for \\ Reproductive Endocrinology of Ministry of Education, Shandong Provincial Key Laboratory of Reproductive Medicine, \\ National Research Center for Assisted Reproductive Technology and Reproductive Genetics, Center for Reproductive \\ Medicine, Shandong Provincial Hospital, Shandong University, Jinan, China
}

OPEN ACCESS

Edited by:

Michael Von Wolff, Universitätsspital Bern, Switzerland

Reviewed by:

Jürgen Michael Weiss, University of Lucerne, Switzerland Yodo Sugishita, St. Marianna University School of Medicine, Japan

*Correspondence: Yanzhi Du

duyz@sjtu.edu.cn

†These authors have contributed equally to this work

Specialty section:

This article was submitted to Reproduction,

a section of the journal

Frontiers in Endocrinology

Received: 01 August 2018

Accepted: 26 October 2018

Published: 15 November 2018

Citation:

Di F, LiU J, Li S, Yao G, Hong Y, Chen Z-J, Li W and Du Y (2018) ATF4 Contributes to Ovulation via Regulating COX2/PGE2 Expression: A

Potential Role of ATF4 in PCOS.

Front. Endocrinol. 9:669.

doi: 10.3389/fendo.2018.00669
Ovulatory disorder is common in patients with hyperprolactinemia or polycystic ovary syndrome (PCOS). Previous studies have shown that ATF4 plays critical role in apoptosis and glucose homeostasis, but its role in regulating reproductive function was not explored. The present study investigated the role of ATF4 in ovarian ovulatory function. Human granulosa cells (hGCs) from 48 women newly diagnosed with PCOS and 37 controls were used to determine ATF4 expression. In vitro cultured hGCs were used to detect the upstream and downstream genes of ATF4. A shRNA- Atf4 lentiviral vector (shAtf4) was injected into rat ovaries to establish an in vivo gene knockdown model to further assess the in vivo relevance of the results from PCOS women. We found that ATF4 expression was lower in hGCs from PCOS patients than in hGCs from non-PCOS women. Many pivotal transcripts involved in cumulus-oocyte complex (COC) expansion, extracellular matrix (ECM) remodeling, and progesterone production were significantly down-regulated after ATF4 knockdown. ChIP-qPCR assays indicated that ATF4 could directly bind to the COX2 promoter and that ATF4 knockdown could attenuate human chorionic gonadotropin (hCG)-induced COX2 expression and PGE2 production. The in vivo study showed that shRNA-lentivirus mediated Atf4 knockdown in rat ovaries led to reduced number of retrieved oocytes. Collectively, these findings suggested previously unknown roles of ATF4 in ovulation. Furthermore, ATF4 malfunction in PCOS patients may impact the ovulation process, which could contribute, in part, to the pathogenesis of PCOS.

\section{Keywords: ATF4, ovulation, human granulosa cells, COX2, polycystic ovary syndrome}

\section{PRÉCIS}

ATF4 in hGCs of PCOS patients was decreased, which impeded hCG-induced COX2 expression and PGE2 production by reducing transcriptional activation and reduced the number of retrieved oocytes in rats.

\section{INTRODUCTION}

PCOS, also called Stein-Leventhal syndrome, is an endocrine disorder characterized by oligoovulation or continuous anovulation, insulin resistance, and hyperandrogenism. PCOS is common in adolescent and reproductive women and has a morbidity of $9-18 \%(1,2)$. Previous studies have 
verified that multiple genes correlated with hormone biosynthesis and ovulation are significantly altered in hGCs $(3,4)$. For the release and transport of oocytes through the oviduct in fertilization, it is essential that the COC matrix is well composed and organized with proper expansion (5). Diminished COC expansion may partially explain the anovulation in women with PCOS (6). However, the molecular mechanism of reduced ovulation in PCOS remains unclear.

Activating transcription factor 4 (ATF4), also known as CREB2, is constitutively expressed in a wide variety of tissues (7). Accumulating evidence suggests that ATF4 plays an important role in regulating the high-level proliferation that is required during osteoblast differentiation (8). ATF4 is able to form heterodimers with members of C/EBP families, including $\mathrm{C} / \mathrm{EBP} \alpha$ and $\mathrm{C} / \mathrm{EBP} \beta$, which are essential for ovulation $(9,10)$. Several studies also identified the role of ATF4 in reproduction. Decreased ATF4 expression in chorionic villus tissue could trigger miscarriage in pregnant women (11). Recently published studies revealed the role of ATF4 in regulating follicular function. For instance, altered expression of ATF4 closely correlated with the development of follicles (12-14). Besides, ATF4 could be induced in the functional and early regression stages of the corpus luteum (CL) (15). However, the specific regulatory mechanisms underlying the function of ATF4 in ovulation remain unclear. The abnormal gene expression profiles of the granulosa cells and ovaries from PCOS patients have revealed many differentially expressed genes $(4,16,17)$. Utilizing bioinformatics methods (DAVID Bioinformatics Resources 6.7), we screened for transcription factors that can trigger differentially expressed genes (data not shown). Among those transcript factors, ATF4 was singled-out due to high frequency of its occurrence in the gene expression profiles. Therefore, based on the published microarray data, we hypothesized that ATF4 may play a critical role in PCOS patients.

Cyclooxygenase-2 (COX2) is the key enzyme responsible for the conversion of arachidonic acid into prostaglandins, among which prostaglandin E2 (PGE2) plays important role in multiple physiological and pathological events. hCG can induce COX2 expression and PGE2 production $(18,19)$. It was reported that COX2-deficient mice were susceptible to reproductive malfunction, such as ovulation disorder, decreased fertilization rate, and embryo implantation dysfunction. Thus, COX2 and PGE2 are markers for evaluating ovulation function (20). A previous study revealed that ATF4 could up-regulate COX2 expression in the kidneys $(21,22)$. These findings emphasized a close correlation between ATF4 and COX2. ATF4 is also known to participate in $\mathrm{HBx}$-mediated COX2 induction via binding to the COX2 promoter (23). These findings strongly indicated that

\footnotetext{
Abbreviations: ATF4, activating transcription factor 4; ChIP, chromatin immunoprecipitation; CL, corpus luteum; COC, cumulus-oocyte complex; COX2, cyclooxygenase-2; DAB, diaminobenzidine; ECM, extracellular matrix; ELISA, enzyme-linked immunosorbent assay; ER, endoplasmic reticulum; FF, follicular fluid; hCG, human chorionic gonadotropin; hGCs, human granulosa cells; IVFET, in vitro fertilization-embryo transfer; LH, luteinizing hormone; OD260, optical density at $260 \mathrm{~nm}$; PCOS, polycystic ovary syndrome; PGE2, prostaglandin E2; PTX3, pentraxin 3 .
}

ATF4, the upstream regulator of COX2, may play a pivotal role in fertilization.

\section{MATERIALS AND METHODS}

\section{Recruitment of Patients}

Eighty five female participants were randomly recruited from the Center for Reproductive Medicine, Ren Ji Hospital, School of Medicine, Shanghai Jiao Tong University, between September 2016 and September 2017. Forty eight of the participants, who were between 20 and 35 years old, were diagnosed with PCOS according to the Rotterdam criteria (oligo- and/or anovulation; clinical and/or biochemical signs of hyperandrogenism; and polycystic ovaries with the exclusion of other causes of hyperandrogenism, such as hyperprolactinemia, androgen-secreting tumors, Cushing's syndrome, and nonclassical congenital adrenal hyperplasia) and received in vitro fertilization-embryo transfer (IVF-ET) (24). The diagnosis of PCOS was satisfied when two or more of the three criteria were met. The remaining 37 participants in the nonPCOS group were healthy females with regular menstrual cycles (26-35 days) and normal ovarian morphology, and were recruited during visits for routine physical examination, tubal factor infertility, or husband's infertility. Endocrine parameters were measured for the non-PCOS women to exclude hyperandrogenism. None of the participants had received hormonal therapy for at least 3 months before the study. All subjects were of Han ethnicity and underwent gonadotrophinreleasing hormone agonist ( $\mathrm{GnRHa}$ ) protocols. After adequate follicle development, hCG (Lvzhu, China) was administered to trigger ovulation. Oocyte retrieval was performed at $36 \mathrm{~h}$ after hCG administration. The basal serum hormonal profiles including FSH, LH, Testosterone (T), estradiol (E2) and prolactin (PRL) were determined using chemiluminescence assay kits

TABLE 1 | Biochemical indexes from controls and PCOS patients.

\begin{tabular}{lcc}
\hline & Control $(\boldsymbol{n}=\mathbf{3 7})$ & PCOS $(\boldsymbol{n}=\mathbf{4 8})$ \\
\hline Age $($ year $)$ & $27.53 \pm 1.32$ & $26.32 \pm 0.96$ \\
BMI $\left(\mathrm{kg} / \mathrm{m}^{2}\right)$ & $21.32 \pm 0.82$ & $22.29 \pm 1.39$ \\
Basal FSH $(\mathrm{IU} / \mathrm{L})$ & $7.19 \pm 0.57$ & $6.94 \pm 0.42$ \\
Basal LH $(\mathrm{IU} / \mathrm{L})$ & $4.73 \pm 0.49$ & $7.54 \pm 1.37^{\star}$ \\
Basal E2 $(\mathrm{pg} / \mathrm{mL})$ & $43.48 \pm 5.70$ & $48.54 \pm 10.76$ \\
Basal T $(\mathrm{nmol} / \mathrm{mL})$ & $1.08 \pm 0.23$ & $1.42 \pm 0.22^{\star}$ \\
Basal PRL $(\mu \mathrm{g} / \mathrm{L})$ & $13.80 \pm 2.07$ & $15.86 \pm 2.53$ \\
LH/FSH & $0.74 \pm 0.23$ & $1.66 \pm 0.14^{*}$ \\
AMH $(\mathrm{ng} / \mathrm{mL})$ & $5.67 \pm 0.30$ & $10.58 \pm 3.56^{* *}$ \\
AFC & $12.38 \pm 2.43$ & $29.36 \pm 4.64^{* * *}$ \\
FBG $(\mathrm{nmol} / \mathrm{L})$ & $4.84 \pm 0.39$ & $5.02 \pm 0.15$ \\
Number of retrieved oocytes & $13.43 \pm 1.27$ & $16.33 \pm 3.72^{*}$ \\
\hline
\end{tabular}

$E_{2}$, estradiol; T, testosterone; PRL, prolactin; $A M H$, Anti-Müllerian Hormone; FBG, fasting blood glucose;

Data are presented as mean \pm SEM. ${ }^{*} P<0.05,{ }^{* \star} P<0.01$ and ${ }^{* \star} P<0.001$ vs. control group. 
(Roche Diagnosis Mannheim, Germany) on the Cobas 6000 analyzer (Roche). Anti-Mullerian hormone (AMH) assay was detected using an ultrasensitive two-site ELISA (AnshLabs, USA) following the manufacturer's protocol. USG was done for the antral follicle count (AFC). The study was approved by the ART Ethics Committee of Ren Ji Hospital, School of Medicine, Shanghai Jiao Tong University. Written informed consent was obtained from all participants. The clinical characteristics of the PCOS and non-PCOS groups are shown in Table 1.

\section{Cell Culture}

hGCs were collected from the PCOS and non-PCOS subjects after their first IVF/intracytoplasmic sperm injection cycle at our center. At $36 \mathrm{~h}$ after triggering, COCs were retrieved via the transvaginal ultrasound-guided aspiration of follicles $\geq 10 \mathrm{~mm}$. Follicular fluid (FF) from the patients was pooled. The protocol of GC isolation and culture was slightly modified from the previously described method (25). In brief, the FF was immediately centrifuged at $1,000 \mathrm{rpm}$ for $10 \mathrm{mins}$ to avoid postaspiration cell death. The cell pellet was resuspended with $0.01 \%$ phosphate-buffered saline (PBS). The hGCs were purified by density gradient centrifugation with Ficoll-Paque (GE Healthcare Bio-Sciences, UK) and then digested with hyaluronidase (Sigma, USA) at $37^{\circ} \mathrm{C}$ for 7 mins. The dispersed cells were collected and cultured in Dulbecco's modified Eagle medium /Ham's F12 with
$10 \%$ fetal bovine serum (Gibco, USA), $100 \mathrm{U} / \mathrm{mL}$ penicillin and $100 \mathrm{mg} / \mathrm{mL}$ streptomycin sulfate (Invitrogen, USA). The viable cells were seeded at $10^{6}$ cells per well in a six-well culture plate, at $5 \times 10^{5}$ cells per well in a 12 -well culture plate or at $1 \times 10^{4}$ cells per well in a chamber slide. For hCG stimulation, culture medium was replaced with fresh medium supplemented with $10 \mathrm{IU} / \mathrm{mL}$ hCG for $24 \mathrm{~h}$ according to a previous study (26). For the signaling inhibition experiment, an ERK inhibitor (PD98059, Selleckchem, USA), AKT inhibitor (LY294002, Selleckchem) or PKA inhibitor (SQ22536 and H89, Selleckchem) was added for $24 \mathrm{~h}$. The culture medium was changed daily for all experiments. Before treatment, hGCs were cultured in serum-free medium for $72 \mathrm{~h}$.

\section{Immunofluorescence Staining}

On the third day of culture, cells in a chamber slide (BD Biosciences, USA) were fixed in $4 \%$ paraformaldehyde and then permeabilized with $0.4 \%$ Triton X-100. After washing, the cells were blocked with normal goat serum (Proteintech, China) for $1 \mathrm{~h}$ and then incubated with ATF4 (1:200, RRID: AB_205875, Santa Cruz, USA) overnight at $4^{\circ} \mathrm{C}$. After washing with phosphate-buffered saline, Alexa Fluor 594 goat anti-mouse immunoglobulin G (red, 1:200, Proteintech, China) was used as secondary antibodies, and the cells were incubated in the dark for $2 \mathrm{~h}$. The nuclei were counterstained with $4^{\prime}, 6^{\prime}$-diamidino2-phenylindole (blue). Staining intensity was examined using a fluorescence microscope.

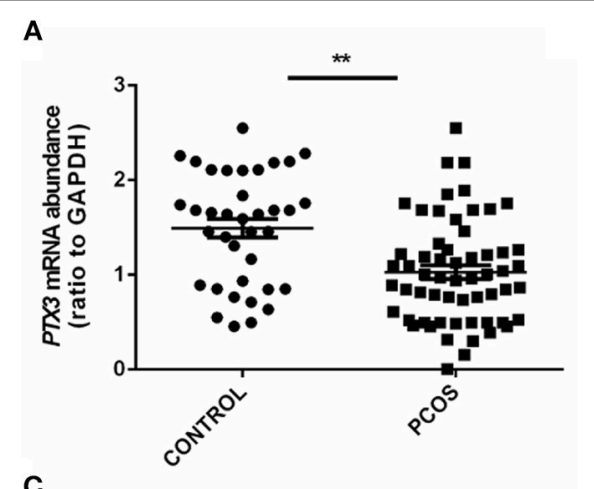

B
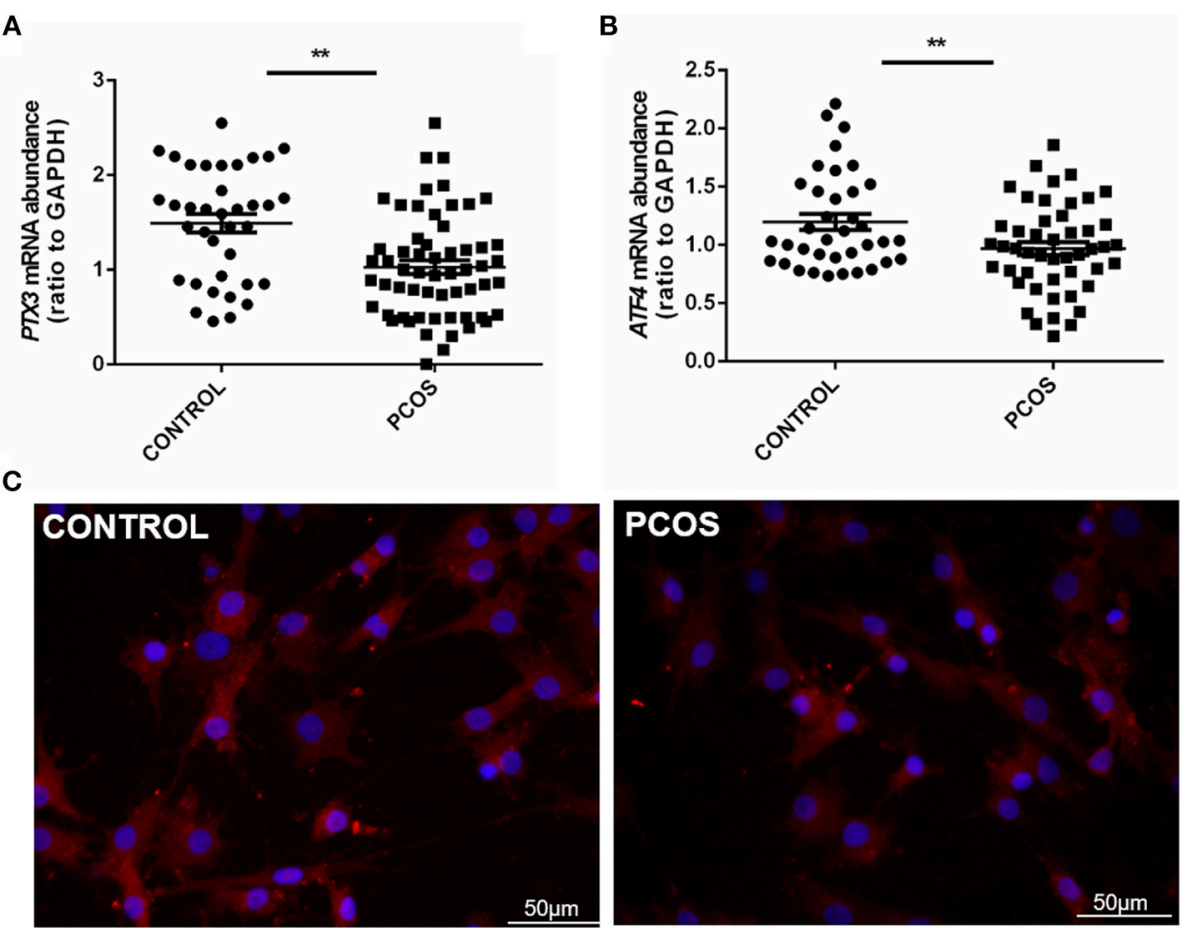

FIGURE 1 | PTX3 and ATF4 expression in hGCs from women with PCOS was decreased. (A,B) Primary hGCs were isolated from healthy controls ( $n=37$ ) and PCOS patients $(n=48)$. PTX3 and ATF4 mRNA levels in primary hGCs were analyzed by qRT-PCR. ${ }^{*} P<0.01$ vs. the control group. (C) The representative images of immunofluorescence staining of ATF4 were performed in a non-PCOS and a PCOS patient. ATF4 (red) is positively expressed in hGCs. Cellular nuclei (blue) were stained with 40, 6-diamidino-2-phenylindole (DAPI). 


\section{Transfection Experiment}

$2 \times 10^{5}$ hGCs were transfected with $50 \mathrm{nM}$ siRNA (GenePharma, China) in Opti-MEM (Invitrogen). The nucleotide sequences for ATF4 siRNA were as follows: sense $5^{\prime}$ - CUCCCAGAAAGUUUAACAATT-3'; antisense: 5'- UUGUUAAACUUUCUGGGAGTT-3'. The cells were electroporated at $175 \mathrm{~V}$ for $5 \mathrm{~ms}$ using an NEPA21 electroporator, for transfection. After dilution with Dulbecco's modified Eagle's medium/F12 containing $10 \%$ fetal bovine serum and antibiotics, the cells were transferred to a six-well culture plate and were ready for treatment after $72 \mathrm{~h}$ of incubation. The knockdown efficiency was determined using quantitative real-time polymerase chain reaction (qRT-PCR) or Western blot assays.

\section{qRT-PCR}

Total RNA was extracted from hGCs using a total RNA isolation kit (FOREGENE, China), the samples were then stored at $-80^{\circ} \mathrm{C}$ for subsequent analysis. RNA concentrations in each sample were determined by calculating the OD260/OD280 ratio using an ultramicro spectrophotometer (Thermo Fisher Scientific, USA). A total of $500 \mathrm{ng}$ of RNA was used for cDNA synthesis with the PrimeScript RT Master Mix Perfect RealTime Kit (TaKaRa, China). Three separate experiments were performed, and each sample was assayed in triplicate. The mean value was used for the determination of mRNA levels by the comparative $\mathrm{Ct}$
$\left(2^{-\Delta \Delta \mathrm{Ct}}\right)$ method, with GAPDH as the reference gene. The primer sequences are shown in the Supplemental Table 1.

\section{Western Blot Analysis}

Total protein was extracted from ovarian tissues, mixed with sodium dodecyl sulfate (SDS) sample buffer, and then boiled for 10 mins. For protein analyses, $30 \mu \mathrm{g}$ of protein from each sample was loaded onto an SDS polyacrylamide gel for electrophoresis and subsequently transferred onto polyvinylidene fluoride membranes. The membranes were blocked in 5\% skim milk for 60 mins at room temperature before incubation overnight at $4^{\circ} \mathrm{C}$ with the following primary antibodies: anti-ATF4 (1:1,000, Santa Cruz, USA, RRID: AB_2058752), anti-COX2 (1:1,000, Proteintech, China, RRID: AB_2085127), and anti-GAPDH (1:5,000, Abcam, USA, RRID: AB_2107448). The samples were then incubated for 90 mins at room temperature with secondary antibodies (1:5,000, Cell Signaling, USA). Chemiluminescence reagent (Thermo Fisher Scientific, USA) was used to visualize the blots. The representative blots were obtained from three independent experiments.

\section{Enzyme-Linked Immunosorbent Assay (ELISA) for PGE2}

A human PGE2-specific ELISA kit was used in accordance with the manufacturer's protocol (Cayman Chemical, USA). Culture medium was collected, and the PGE2 levels in the
A

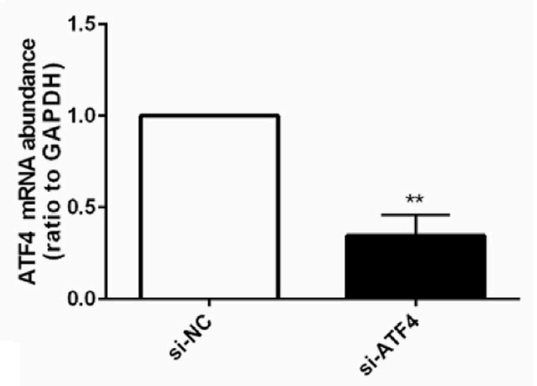

C

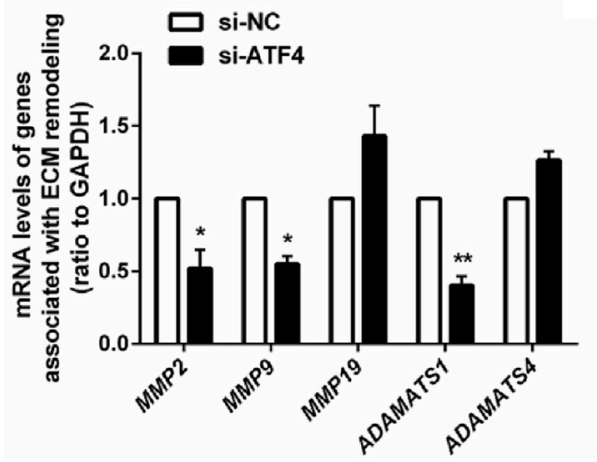

B

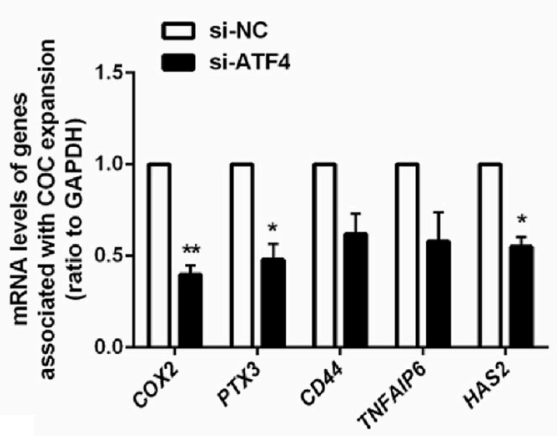

D

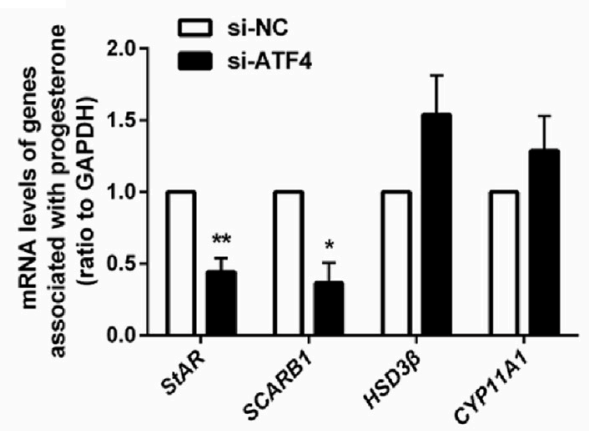

FIGURE 2 | ATF4 in hGCs regulated a variety of genes associated with ovulatory response. (A) $2 \times 10^{5}$ hGCs were transfected with $50 \mathrm{nM}$ control siRNA (si-NC) or ATF4 siRNA (si-ATF4) for $48 \mathrm{~h}$. The efficacy of siRNA-mediated ATF4 knockdown was examined in hGCs. (B-D) The expression levels of genes related to COC expansion, ECM remodeling and progesterone synthesis were analyzed by qRT-PCR. ${ }^{\star} P<0.05$ and ${ }^{\star \star} P<0.01$ vs. the si-NC group. 
A

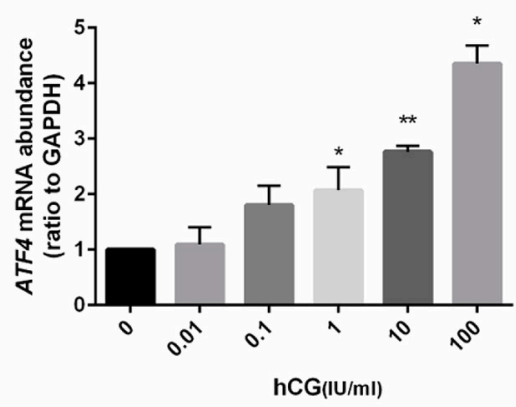

C
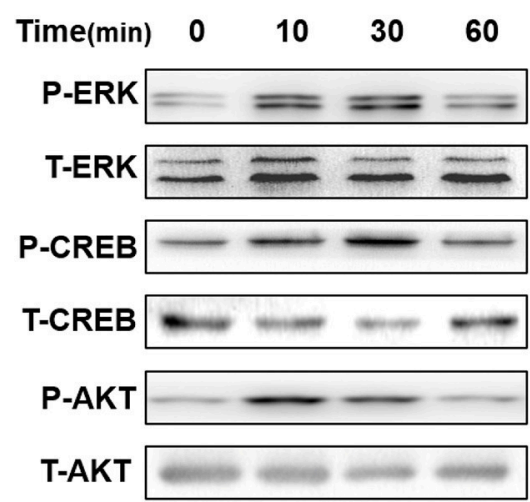

E

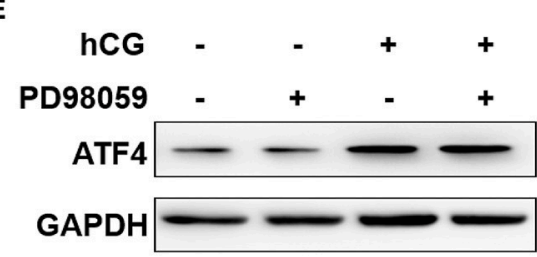

G

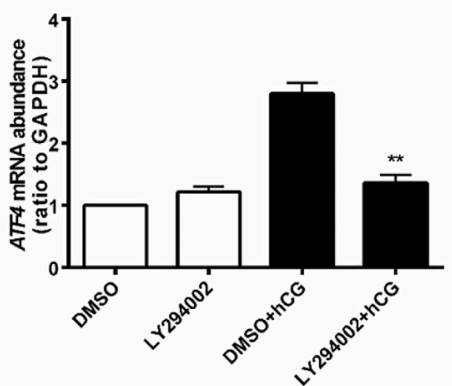

B

$\begin{array}{lllllll}\text { hCG } & 0 & 0.01 & 0.1 & 1 & 10 & 100\end{array}$

ATF4 $m-\infty m$

GAPDH $-\cdots \cdots$

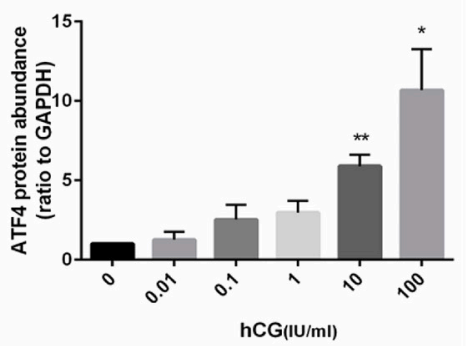

D

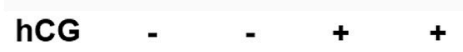

SQ22536 - + $\quad+\quad+$
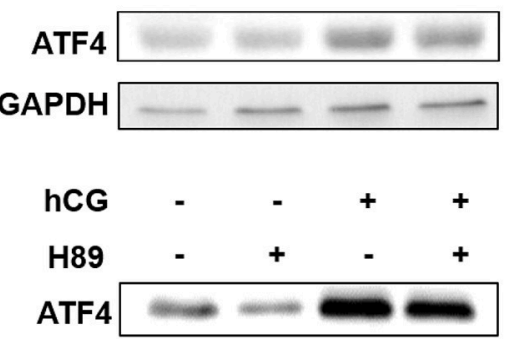

GAPDH $--\cdots$
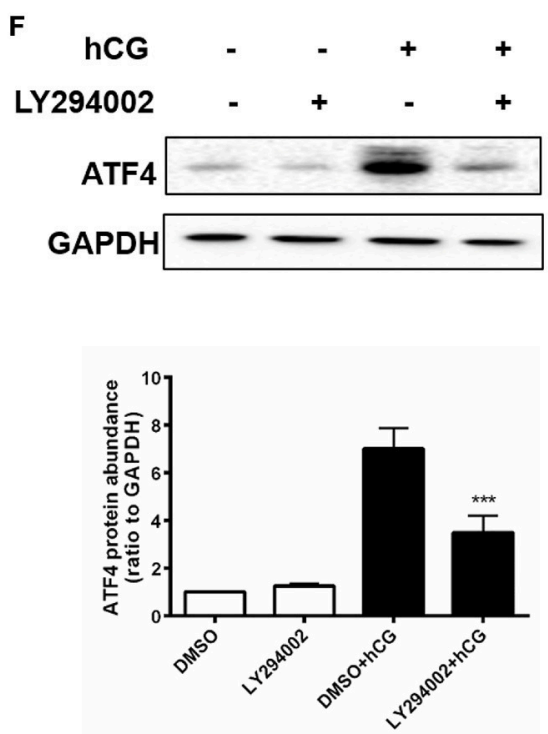

FIGURE 3 | AKT signaling was required for hCG-induced ATF4 expression in hGCs. (A,B) Cells were treated with 0, 0.01, 0.1, 1, 10, and 100 IU/mL hCG for $24 \mathrm{~h}$ as indicated. ATF4 mRNA and protein expression levels were analyzed in hGCs. ${ }^{*} P<0.05$ and ${ }^{*} P<0.01$ vs. the $0 \mathrm{IU} / \mathrm{mL}$ group. (C) Cells were treated with $10 \mathrm{IU} / \mathrm{mL}$ hCG for 0, 10, 30, or 60 mins. CREB, ERK1/2, and AKT phosphorylation levels were examined by western blot. (D,E) Cells were pre-treated with DMSO (vehicle control), $10 \mu \mathrm{M}$ SQ22536, $10 \mu \mathrm{M} \mathrm{H89}$, or $10 \mu \mathrm{M}$ PD98059 for $1 \mathrm{~h}$ and then treated with DMSO or $10 \mathrm{IU} / \mathrm{mL} \mathrm{hCG}$ for $24 \mathrm{~h}$. ATF4 protein expression levels in hGCs were determined by western blot. (F,G) Cells were pre-treated with DMSO or $10 \mu \mathrm{M} \mathrm{LY} 294002$ for $1 \mathrm{~h}$ and then treated with DMSO or $10 \mathrm{IU} / \mathrm{mL} \mathrm{hCG}$ for $24 \mathrm{~h}$. ATF4 expression in hGCs was determined by qRT-PCR and western blot. ${ }^{* *} P<0.01$ and ${ }^{* \star *} P<0.001$ vs. the DMSO + hCG group. 
A

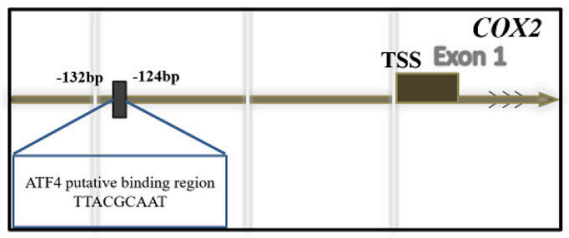

B

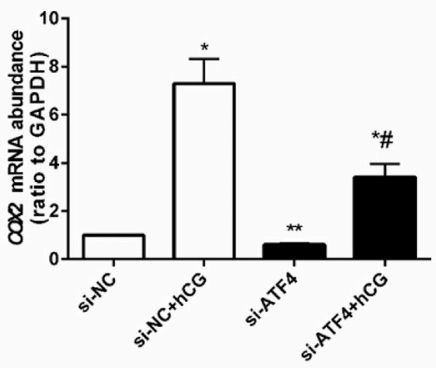

D

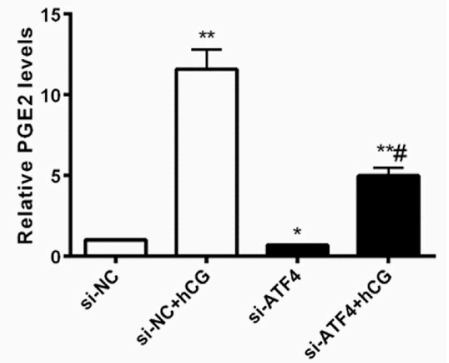

E

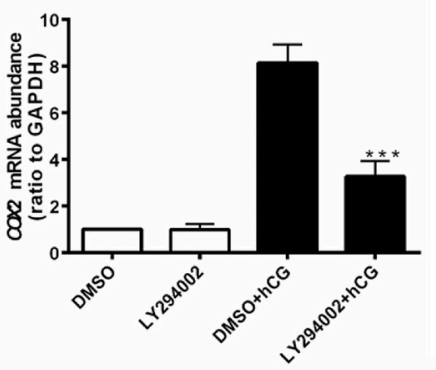

G

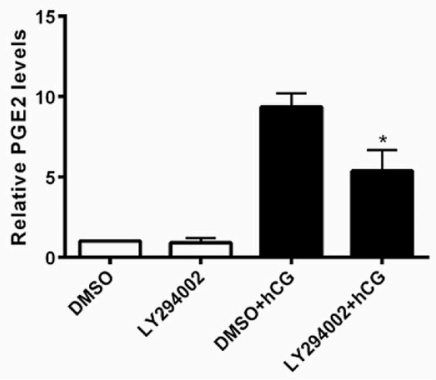

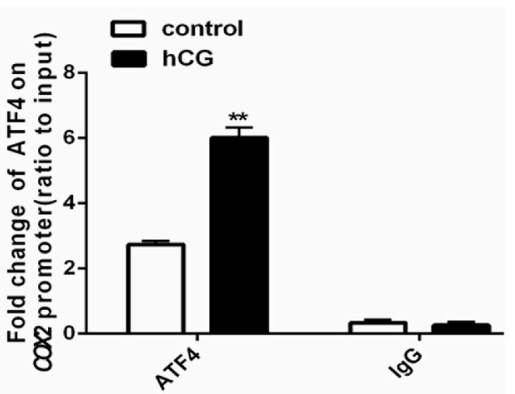

C
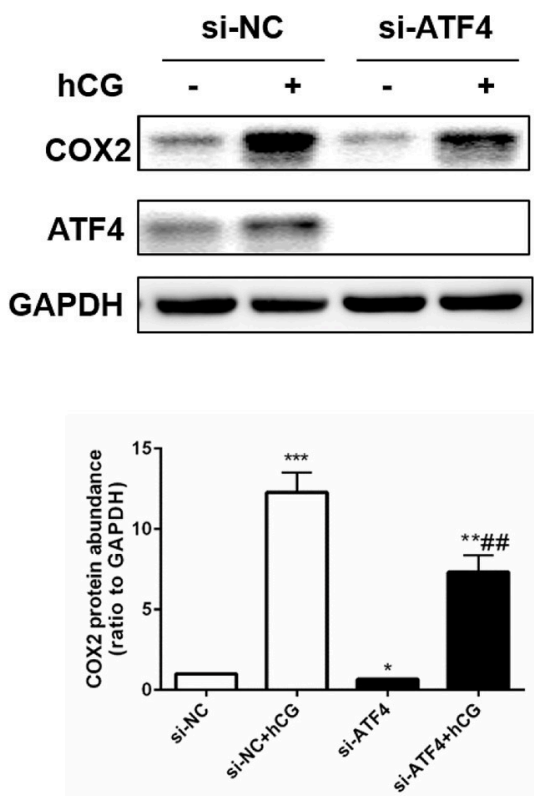

F
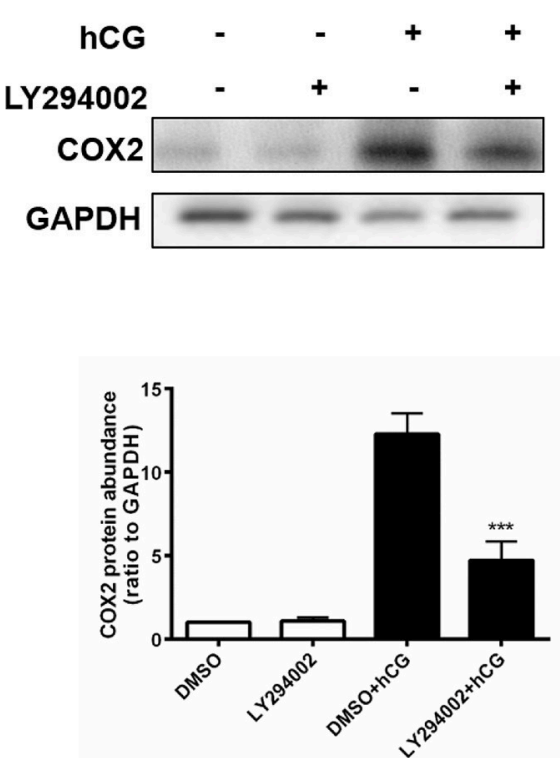

FIGURE 4 | ATF4 could directly bind to the COX2 promoter, and ATF4 knockdown attenuated hCG-induced COX2 expression and PGE2 production in hGCs.

(A) Cells were treated with DMSO or $10 \mathrm{IU} / \mathrm{mL}$ hCG for $24 \mathrm{~h}$. Normalized inputs of chromatin DNA from hGCs were pulled down with ATF4 or negative IgG antibodies. 
FIGURE 4 | DNA templates were amplified by PCR. ${ }^{\star \star} P<0.01$ vs. the DMSO group. (B) Cells were transfected with $50 \mathrm{nM}$ si-NC or si-ATF4 for $48 \mathrm{~h}$ and then treated with DMSO or $10 \mathrm{IJ} / \mathrm{mL}$ hCG for $24 \mathrm{~h}$. COX2 mRNA levels were detected by qRT-PCR analysis. (C) COX2 and ATF4 protein levels were detected by western blot analysis. (D) PGE2 levels in the culture supernatants were measured by ELISA. ${ }^{\star} P<0.05,{ }^{* \star} P<0.01$, and ${ }^{\star \star *} P<0.001$ vs. the si-NC group, $\# P<0.05$ and $\# \# P<$ 0.01 vs. the si-NC + hCG group. (E) Cells were pre-treated with DMSO or $10 \mu \mathrm{M} \mathrm{LY} 294002$ for $1 \mathrm{~h}$ and then treated with DMSO or $10 \mathrm{IU} / \mathrm{mL} \mathrm{hCG}$ for $24 \mathrm{~h}$. COX2 mRNA levels were detected by qRT-PCR analysis. (F) COX2 protein levels were detected by western blot analysis. (G) PGE2 levels in the culture supernatants were measured by ELISA. ${ }^{*} P<0.05$ and ${ }^{* \star *} P<0.001$ vs. the DMSO + hCG group.

culture medium were determined by ELISA kit. The OD values for PGE2 were normalized with the protein concentrations of the corresponding cell lysates. The normalized PGE2 values obtained from the treated cells are relative to those of the control cells. The linear range of the PGE2 concentrations was $7.8-1,000 \mathrm{pg} / \mathrm{mL}$.

\section{Chromatin Immunoprecipitation (ChIP) Assay}

ChIP assays were performed as described by the manufacturer (Upstate Biotechnology, NY) with some modifications. Briefly, hGCs were cultured with or without $10 \mathrm{IU} / \mathrm{ml}$ hCG for $24 \mathrm{~h}$. Chromatin solutions were sonicated, incubated with an anti-ATF4 antibody or control IgG, and rotated overnight at $4^{\circ} \mathrm{C}$. Then, the enriched chromatin DNA was purified and subjected to PCR analysis. To amplify the human COX2 promoter region containing the ATF4-binding site, the following primer sets were used: COX2 ChIP Forward, 5'-AGCTTCCTGGGTTTCCGATTTTCT-3' and COX2 ChIP Reverse, 5'-CCCTGCTGAGGAGTTCCTGGA-3'.

\section{Animal Studies}

Atf4 -RNAi-Lentivirus was injected intrabursally into 8week-old female Wistar rat ovaries with a $10 \mu$ l-syringe (Gaoge, China). The targeting sequence of the shRNA was 5'- GACAGCTAAAGTGAAGACTGA-3'. The recombinant lentivirus of small interference RNA targeting Atf4 (Atf4 RNAi-Lentivirus) and the control lentivirus (GFP-lentivirus) were prepared and titered to $10^{9} \mathrm{TU} / \mathrm{mL}$ (transfection unit). The needle was inserted slowly and held in place for $5 \mathrm{~min}$. Each ovary was injected twice at different sites with $10 \mu \mathrm{l}$ each time. Ten days after lentivirus injection, each rat was injected intrabursally with 40 IU pregnant mare's serum gonadotropin (PMSG; PROSPEC, USA) to stimulate follicle development for $48 \mathrm{~h}$, then, $10 \mathrm{IU}$ hCG was given to induce ovulation as described before (27). The rats were sacrificed by decapitation at $16 \mathrm{~h}$ after hCG administration. All experimental procedures were approved by the Institutional Review Board of Ren Ji Hospital, School of Medicine, Shanghai Jiao Tong University. All rats received humane care in accordance with the National Institutes of Health Guide for the Care and Use of Laboratory Animals.

\section{Statistical Analysis}

The data are presented as the mean \pm SEM. All analyses were conducted using SPSS 21.0 software for Windows (IBM, USA). All data was tested for normality. Paired group means were compared for statistical significance using Student's $t$-tests, while multiple means were compared by analysis of variance (ANOVA) followed by Tukey's post hoc tests. Values of $P<0.05$ were considered statistically significant.

\section{RESULTS}

\section{The Expression of ATF4 in hGCs From Women With PCOS Was Decreased}

We first determined whether ATF4 expression was changed in clinical PCOS patients. As shown in Table 1, compared with the controls, PCOS patients were characterized by increased testing indexes, including basal LH, LH/FSH, T and AMH levels. qRTPCR was performed to measure ATF4 mRNA in primary hGCs. As shown in Figure 1A, pentraxin 3 (PTX3) levels were lower in the PCOS group than in the non-PCOS group, in accordance with previous research results (28). The mRNA and protein abundance of ATF4 were lower in the PCOS group than in the control group (Figures 1B,C). Therefore, in vitro and in vivo experiments were performed to explore the physiological role of ATF4.

\section{ATF4 Regulated a Variety of Genes Associated With Ovulatory Response in hGCs}

To further illuminate the role of ATF4 in ovulation regulation, siRNA-mediated gene knockdown was used to down-regulate the endogenous expression of ATF4 in hGCs. The qRT-PCR results showed a significant decrease in ATF4 expression in hGCs (Figure 2A). More importantly, we found that several ovulationrelated genes were significantly altered. The mRNA levels of genes associated with COC expansion in hGCs, including COX2, PTX3, CD44, TNFAIP6, and HAS2, were decreased when ATF4 was deficient (Figure 2B). Other genes that play a pivotal role in extracellular matrix (ECM) remodeling were detected through qRT-PCR. The mRNA expression of $M M P 2$, $M M P 9$, and ADAMTS1 in hGCs was significantly impeded when ATF4 knockdown was achieved with RNAi (Figure 2C). The mRNA expression levels of genes involved in progesterone synthesis, such as StAR and SCARB1, were also decreased, while the expression levels of $H S D 3 \beta$ and $C Y P 11 A 1$ were barely changed (Figure 2D). Altogether, these results indicated that ATF4 participates in regulating the expression of ovulatory genes.

\section{AKT Signaling Was Required for hCG-induced ATF4 Expression in hGCs}

Given that ATF4 plays such a critical role in regulating genes essential for ovulation, the effects of hCG on ATF4 expression in hGCs were then examined. Cultured hGCs were treated with 
different concentrations of hCG. The results obtained from qRTPCR and Western blot analyses showed that hCG positively regulated the mRNA and protein abundance of ATF4 in hGCs in a dose-dependent manner. In particular, hCG treatment at concentrations higher than $10 \mathrm{IU} / \mathrm{mL}$ significantly induced ATF4 expression in hGCs (Figures 3A,B). These results collectively revealed that ATF4 expression in hGCs was elevated after hCG stimulation. The mechanisms underlying the regulation of ATF4 by hCG remain unclear. Herein, several signaling pathways, including the PKA/CREB, ERK1/2 and PI3K/AKT
A

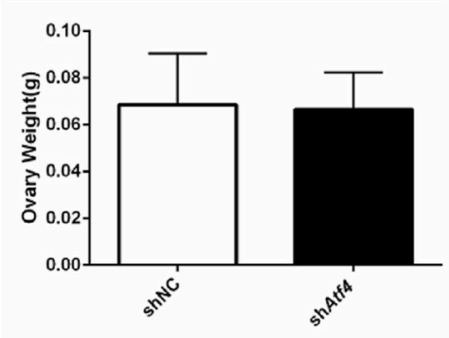

C
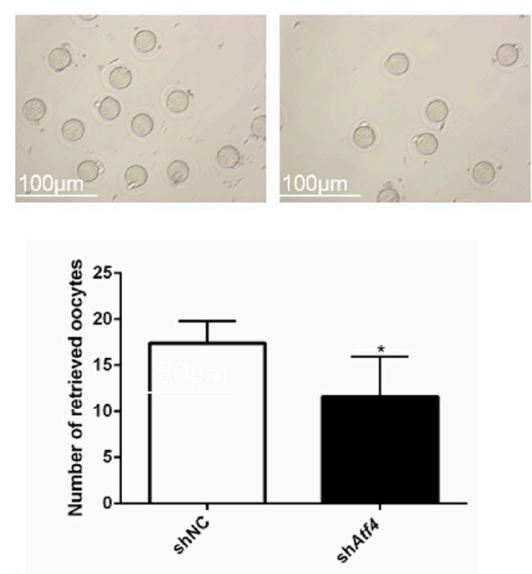

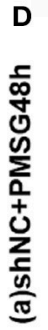
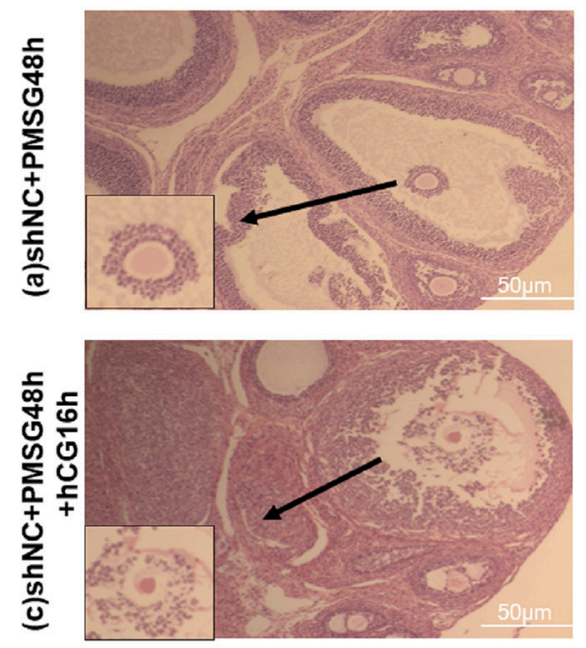

B

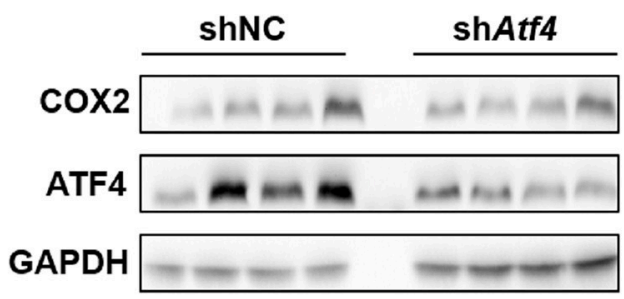

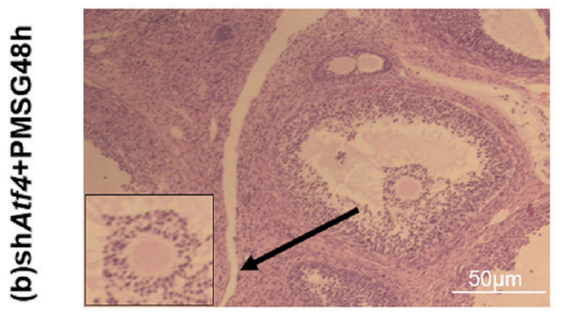

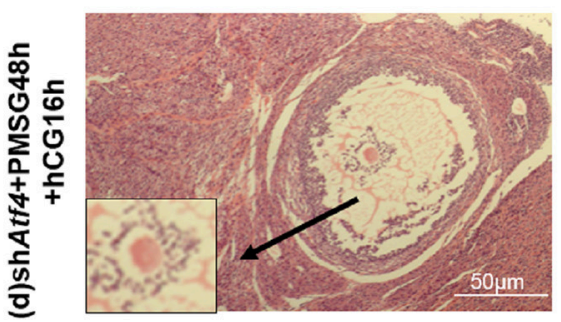

FIGURE 5 | Atf4 in rat ovaries regulates ovulation function. (A) Rats were transfected with lentiviruses encoding shAtf4 or shNC by intrabursal injection. After 7 days, the rats were sacrificed. Ovarian weights were measured. (B) ATF4 and COX2 protein levels in rat ovaries were quantitated by western blot. ${ }^{\star} P<0.05$ vs. shNC. (C) After transfection, the rats were treated with PMSG for $48 \mathrm{~h}$ and then with $\mathrm{hCG}$ for $16 \mathrm{~h}$ for superovulation. The number of oocytes retrieved from the rat fallopian tubes was measured. ${ }^{*} P<0.05$ and ${ }^{* \star} P<0.01$ vs. the shNC group. (D) a,b Hematoxylin stained preovulatory follicles $48 \mathrm{~h}$ was detected after the PMSG treatment (before hCG injection). (D) c,d Hematoxylin stained preovulatory follicles $16 \mathrm{~h}$ was detected after induction of ovulation by hCG injection. 
signaling pathways, that might be involved in this regulation were examined. As shown in Figure 3C, the phosphorylation levels of CREB, ERK1/2, and AKT were increased considerably in hGCs treated with $10 \mathrm{IU} / \mathrm{mL}$ hCG for 10 and $30 \mathrm{~min}$. The data suggested that these signaling pathways were activated under hCG stimulation. To further verify the exact pathway involved in activating ATF4 after hCG stimulation, pharmacological inhibitors were used to block the activation of signaling pathways. Inhibiting CREB by SQ22536 or H89 did not affect the protein expression of ATF4 upon hCG treatment (Figure 3D). Treating cells with PD98059, a specific inhibitor for MEK, did not attenuate the induction of ATF4 expression upon hCG exposure (Figure 3E). Notably, the PI3K inhibitor LY294002 significantly impaired hCG-induced increase in ATF4 expression (Figure 3F). The qRT-PCR results were consistent with the Western blot results (Figure 3G). Taken together, these data indicated that $\mathrm{PI} 3 \mathrm{~K} / \mathrm{AKT}$ signaling pathway activation was required for the hCG-induced increase in ATF4 expression in hGCs.

\section{ATF4 Could Directly Bind to the COX2 Promoter, and ATF4 Knockdown Attenuated hCG-induced COX2 Expression and PGE2 Production in hGCs}

ATF4 can bind to the COX2 promoter (23), and COX2-derived PGE2 plays a critical role in regulating ovulation (29). Thus, we further investigated whether ATF4 directly modulated COX2 by binding to its promoter region in hGCs. We identified a putative ATF4-binding site ( $5^{\prime}$-TTACGCAAT-3') between nucleotides -132 and -124 on COX 2 promoter sequences, by using TESS for predicting transcription-factor-binding sites in DNA sequences(Figure 4A). hGCs were cultured without or with $10 \mathrm{IU} / \mathrm{mL}$ hCG for $24 \mathrm{~h}$. The enriched fragments in the COX2 promoter utilizing an ATF4 antibody were determined through qRT-PCR. The results demonstrated that the fragments to which ATF4 bound in the COX2 promoter were significantly increased in hCG-treated hGCs, suggesting that ATF4 up-regulated COX2 expression mainly by enhancing the transcriptional activity of COX2 via binding to its promoter regions (Figure 4A). As shown in Figures 4B,C, the knockdown of ATF4 by specific siRNA decreased the basal levels of COX2, which was consistent with the results shown in Figure 2B. hCG effectively stimulated the mRNA and protein expression of COX2, while ATF4 knockdown dampened the hCG-induced increase in COX2 abundance (Figures 4B,C). PGE2 followed a similar trend as COX2 (Figure 4D). As shown in Figures 4E-G, the results also showed that hCG increased COX2 expression and PGE2 levels depending on PI3K/AKT pathway activation. To sum up, these data indicated that ATF4 directly bound to the COX2 promoter and that ATF4 knockdown could attenuate hCG-induced COX2 expression and PGE2 production via the PI3K/AKT signaling pathway.

\section{Atf4 in Rat Ovaries Regulates Ovulation Function}

To verify the function of Atf4 in vivo, Atf4-deficient ovarian tissues were established in rats via the intrabursal injection of lentivirus shAtf4. Lentivirus shAtf4 successfully reduced ATF4 expression in rat ovaries without affecting ovary weights (Figures 5A,B). Then, rats were injected with PMSG, followed by hCG injection, $48 \mathrm{~h}$ later. After another $16 \mathrm{~h}$, the rats were sacrificed, and the ovarian tissues and fallopian tubes were harvested. Atf4 knockdown effectively reduced the number of oocytes retrieved from the fallopian tubes (Figure $5 \mathbf{C}$ and Table 2). When shAtf4 rats were treated with PMSG and hCG, preovulatory follicular development was normal (Figure 5D)a,b. However, COC expansion was impaired slightly (Figure 5D)c,d. Furthermore, when COC expansion was examined in vitro, the physiological mediators of COX2 (30), was less effective in inducing expansion of COCs collected from shAtf4 compared with shNC (Figure 5B).The phenomenon of oligo-ovulation in Atf4-deficient rats could be explained by the incorrect expansion of COC. Altogether, these results indicated that Atf 4 was required for the execution of ovulation in rat ovaries.

\section{DISCUSSION}

In the present study, we demonstrated that ATF4 was expressed in hGCs; this finding was indispensable for determining the physiological function of ATF4. Whether ATF4 expression was changed in PCOS patients remained unclear. Thus, we further investigated the role of ATF4 in PCOS by analyzing clinical samples. First, we determined that PTX 3 expression was lower in PCOS, and we regarded this as a positive control result, and this finding was consistent with previous reports (28). Consistently, we found that the levels of ATF4 in primary hGCs from PCOS patients were decreased. These data implied that the aberrant expression of ATF4 might be correlated with PCOS.

The normal expansion of COC is required for ovulation, which requires the abundant expression of transcripts, such as COX2, PTX3, CD44, TNFAIP6, and HAS2 (31). Moreover, variations in the ECM secreted from cumulus cells are also essential for oocyte release. Several classes of proteinases have been reported to regulate ovarian ECM remodeling, including MMPs, the plasmin/plasminogen activator system, and ADAMTS (32). ATF4 has been recently identified as an important controller of reproduction $(13,33)$. The absence of ATF4 in hGCs resulted in a decrease in the expression of multiple genes involved in COC expansion, ECM remodeling, and progesterone production. However, not all markers related to ovulation were downregulated. Ovulation is a highly complex, and primary physiological process that multiple signals involved in. ATF4 could just play important role in this process.

hCG, a hormone secreted by the placenta, can induce progesterone and ovulation production in the ovaries, which

TABLE 2 | Number of retrieved oocytes from rat fallopian tubes.

\begin{tabular}{lcc}
\hline & $\begin{array}{c}\text { shNC group } \\
(\boldsymbol{n}=\mathbf{4})\end{array}$ & $\begin{array}{c}\text { shAtf4 } \\
\text { group }(\boldsymbol{n}=\mathbf{4})\end{array}$ \\
\hline Number of retrieved oocytes & $17.40 \pm 1.077$ & $11.60 \pm 1.939^{*}$ \\
\hline
\end{tabular}

Data are presented as mean $\pm S E M$. ${ }^{\star} P<0.05$ vs. shNC group. 


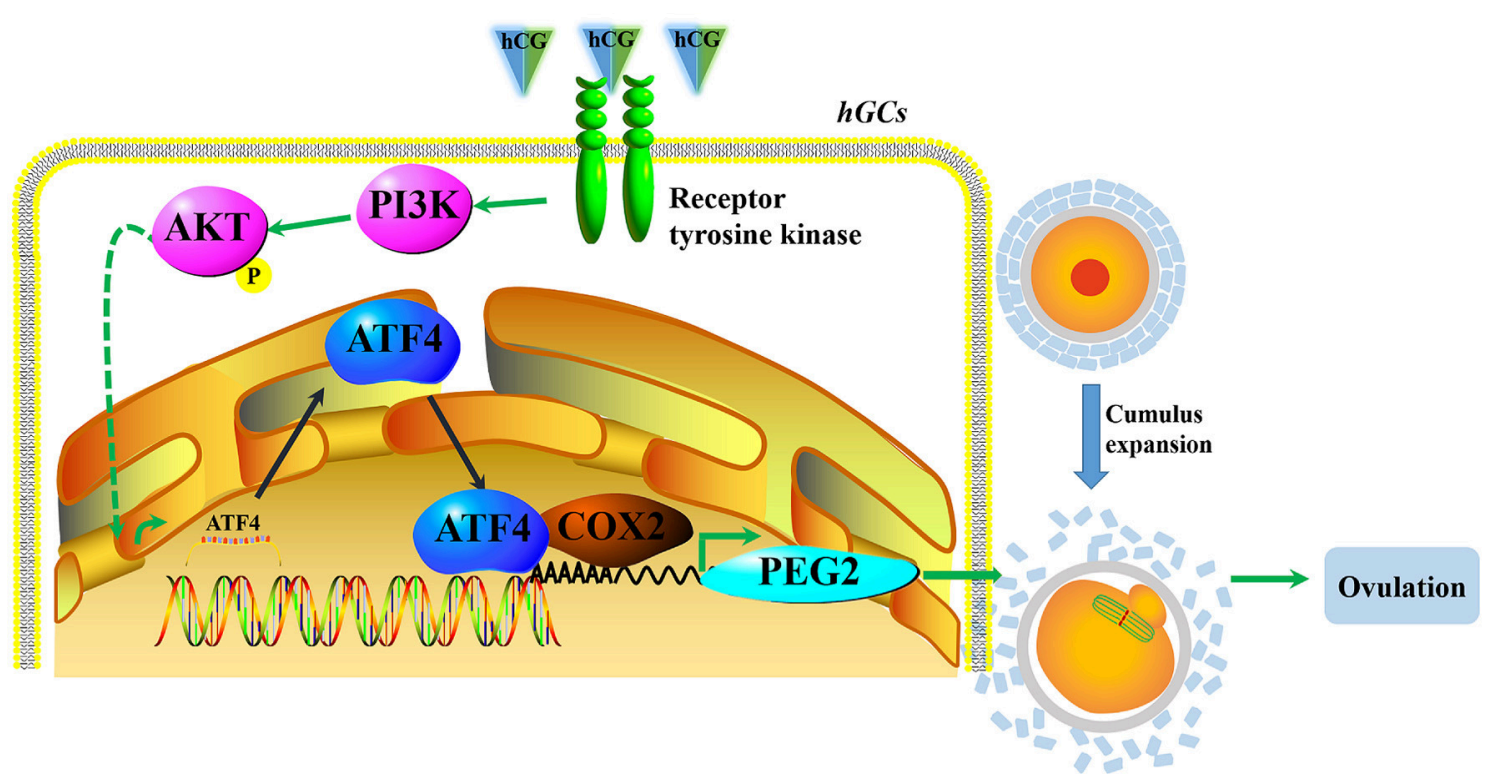

FIGURE 6 | A proposed working model underpinning that decreased ATF4 disrupted ovulation. hCG activated the PI3K/AKT signaling pathway and then stimulated ATF4 expression in hGCs. The increased ATF4 transcripts directly bound to the promoter of COX2, which enhanced the transcription of COX2 and the synthesis of PGE2 to consequently facilitate COC expansion and ovulation.

is consequentially used for the treatment of infertility $(12,34)$. Given that ATF4 plays such a critical role in the ovulation process, the effects of hCG on ATF4 expression in hGCs were then tested. Our subsequent results showed that hCG significantly induced ATF4 expression in hGCs in a concentration-dependent manner. The mechanisms underlying the regulation of ATF4 by hCG are not known. In the present work, several signaling pathways that might be involved in this process were examined. We found that hCG-induced ATF4 up-regulation was related to activation of the PI3K/AKT pathway rather than the PKA/CREB and ERK1/2 signaling pathways.

ATF4 can induce COX2 expression in the kidney, emphasizing the potential correlation between ATF4 and COX2 $(21,22)$, some studies indicate ATF4 induces ovarian granulosa cell damage and inhibits ovarian follicle activation $(13,35)$. Previously, we identified the key role of ATF4 in modulating the expression of COX2 in hGCs (Figure 2B). Consistently, hCG could also induce COX2 expression $(18,19)$. The increases in COX2 and PGE2 levels are representative indicators of ovulation $(36,37)$. Mechanistically, we found that ATF4 deficiency impaired the hCG-mediated induction of COX2 and PGE2 expression and ATF4 likely exerts its effects by directly binding to the promoter of COX2 in hGCs. ATF4 could form heterodimers with members of $\mathrm{C} / \mathrm{EBP}$ families, including $\mathrm{C} / \mathrm{EBP} \alpha$ and $\mathrm{C} / \mathrm{EBP} \beta$, which are essential for ovulation $(9,10)$. The putative ATF4 binding region at the COX 2 promoter could be partially overlapping with the $\mathrm{C} / \mathrm{EBP}$ families, and more subjects should be further required to prove our concluded results. Thus, these findings suggested that ATF4 is closely involved in the regulation of ovulatory indicators.

An in vivo study was also performed to investigate the role of Atf 4 in ovulation. Due to the high efficacy and long-term stability of shRNA, we employed lentivirus shAtf4, which could stably reduce the expression of Atf4 in rat ovaries. Intrabursal injection is a promising method for ensuring the local knockdown of target genes with high efficacy. Compared to the mock transfection, Atf4 knockdown considerably reduced the number of oocytes retrieved from rat fallopian tubes. These results collectively support the pivotal role of Atf4 in maintaining normal ovulation in rats.

In conclusion, our results demonstrate that hCG could activate the PI3K/AKT signaling pathway and then stimulate ATF4 expression in hGCs. The increased ATF4 transcripts directly bound to the promoter of COX2, which enhanced the transcription of COX2 and the synthesis of PGE2 to consequently facilitate COC expansion and ovulation (Figure 6). Moreover, decreased ATF4 expression in hGCs is correlated with PCOS. In vivo, we confirmed that the knockdown of Atf4 reduced ovulation and COC expansion which could be related to the ovulation disorder of PCOS. Our study provides a novel insight into the mechanisms underlying the formation and development of PCOS. In this case, ATF4 could likely be used as a potential target for the treatment of PCOS in the future.

\section{AUTHOR CONTRIBUTIONS}

FD and YD designed the research. FD, SL, and YH collected the clinical patients' samples. FD, JL, SL, and GY performed the experiments. FD, JL, WL, and YD analyzed the data. FD, Z-JC, and YD wrote the article.

\section{FUNDING}

This research was supported by grants from the National Natural Science Foundation (81671414, 81490743 and 81671413), 
the National Key Research and Development Program of China (2017YFC1001002), Shanghai Municipal Education Commission-Gaofeng Clinical Medicine (20152510), and Shanghai Key Laboratory for Assisted Reproduction and Reproductive Genetics (17DZ2271100).

\section{ACKNOWLEDGMENTS}

We thank all of the research volunteers for participating in this study, and we thank the clinicians and embryologists

\section{REFERENCES}

1. Wei Z, Cao Y, Cong L, Zhou P, Zhang Z, Li J. Effect of metformin pretreatment on pregnancy outcome of in vitro matured oocytes retrieved from women with polycystic ovary syndrome. Fertil Steril. (2008) 90:1149-54. doi: 10.1016/j.fertnstert.2007.07.1385

2. Azziz R, Woods KS, Reyna R, Key TJ, Knochenhauer ES, Yildiz BO. The prevalence and features of the polycystic ovary syndrome in an unselected population. J Clin Endocrinol Metab. (2004) 89:2745-9. doi: 10.1210/jc.2003-032046

3. Aydos A, Gurel A, Oztemur Islakoglu Y, Noyan S, Gokce B, Ecemis T, et al. Identification of polycystic ovary syndrome (PCOS) specific genes in cumulus and mural granulosa cells. PLoS ONE (2016) 11:e0168875. doi: 10.1371/journal.pone.0168875

4. Kaur S, Archer KJ, Devi MG, Kriplani A, Strauss JF III, Singh R. Differential gene expression in granulosa cells from polycystic ovary syndrome patients with and without insulin resistance: identification of susceptibility gene sets through network analysis. J Clin Endocrinol Metabol. (2012) 97:E2016-2021. doi: 10.1210/jc.2011-3441

5. Fülöp C, Szántó S, Mukhopadhyay D, Bárdos T, Kamath RV, Rugg MS, et al. Impaired cumulus mucification and female sterility in tumor necrosis factor-induced protein-6 deficient mice. Development (2003) 130:2253-61.

6. Ambekar AS, Kelkar DS, Pinto SM, Sharma R, Hinduja I, Zaveri K., et al. Proteomics of follicular fluid from women with polycystic ovary syndrome suggests molecular defects in follicular development. J Clin Endocrinol Metabol. (2015) 100:744-53. doi: 10.1210/jc.2014-2086

7. Mamady H, Storey KB. Coping with the stress: expression of ATF4, ATF6, and downstream targets in organs of hibernating ground squirrels. Arch Biochem Biophys. (2008) 477:77-85. doi: 10.1016/j.abb.2008.05.006

8. Wortel IMN, van der Meer LT, Kilberg MS, van Leeuwen FN. Surviving stress: modulation of ATF4-mediated stress responses in normal and malignant cells. Trends Endocrinol Metabol. (2017) 28:794-806. doi: 10.1016/j.tem.2017.07.003

9. Vallejo M, Ron D, Miller CP, Habener JF. C/ATF, a member of the activating transcription factor family of DNA-binding proteins, dimerizes with CAAT/enhancer-binding proteins and directs their binding to cAMP response elements. Proc Natl Acad Sci USA. (1993) 90:4679-83.

10. Fan HY, Liu Z, Johnson PF, Richards JS. CCAAT/enhancer-binding proteins (C/EBP) $-\alpha$ and $-\beta$ are essential for ovulation, luteinization, and the expression of key target genes. Mol Endocrinol. (2011) 25:253-68. doi: 10.1210/me.2010-0318

11. Chai L, Ling K, He X, Yang R. Expression of ATF4 and VEGF in chorionic villus tissue in early spontaneous abortion. Eur J Obstet Gynecol Reprod Biol. (2013) 170:434-8. doi: 10.1016/j.ejogrb.2013.07.005

12. Cui Y, Ma Z, Zhao H, Chen X, Zhang Y, Guo H., et al. Activation of eIF2alpha signaling cascade is associated with testosterone-induced cell apoptosis in INS-1 cells. Horm Metab Res. (2014) 46:574-80. doi: 10.1055/s-0034-1374588

13. Barilovits SJ, Newsom KJ, Bickford JS, Beachy DE, Rhoton-Vlasak A, Nick HS. Characterization of a mechanism to inhibit ovarian follicle activation. Fertil Steril. (2014) 101:1450-7. doi: 10.1016/j.fertnstert.2014.01.025

14. Lin P, Yang Y, Li X, Chen F, Cui C, Hu L., et al. Endoplasmic reticulum stress is involved in granulosa cell apoptosis during follicular atresia in goat ovaries. Mol Reprod Dev. (2012) 79:423-32. doi: 10.1002/mrd.22045 of the Reproduction Center of Ren Ji Hospital for their excellent assistance. This manuscript has been proofread and edited by a professional English editing company, Enago.

\section{SUPPLEMENTARY MATERIAL}

The Supplementary Material for this article can be found online at: https://www.frontiersin.org/articles/10.3389/fendo. 2018.00669/full\#supplementary-material

15. Park HJ, Park SJ, Koo DB, Lee SR, Kong IK, Ryoo JW., et al Progesterone production is affected by unfolded protein response (UPR) signaling during the luteal phase in mice. Life Sci. (2014) 113:60-7. doi: 10.1016/j.lfs.2014.07.033

16. Kenigsberg S, Bentov Y, Chalifa-Caspi V, Potashnik G, Ofir R, Birk OS Gene expression microarray profiles of cumulus cells in lean and overweightobese polycystic ovary syndrome patients. Mol Hum Rep. (2009) 15:89-103. doi: 10.1093/molehr/gan082

17. Jansen E, Laven JS, Dommerholt HB, Polman J, van Rijt C, van den Hurk C., et al. Abnormal gene expression profiles in human ovaries from polycystic ovary syndrome patients. Mol Endocrinol. (2004) 18:3050-63. doi: 10.1210/me.2004-0074

18. Duffy DM, Seachord CL, Dozier BL. An ovulatory gonadotropin stimulus increases cytosolic phospholipase A2 expression and activity in granulosa cells of primate periovulatory follicles. J Clin Endocrinol Metab. (2005) 90:5858-65. doi: 10.1210/jc.2005-0980

19. Choi Y, Wilson K, Hannon PR, Rosewell KL, Brännström M, Akin JW, et al. Coordinated regulation among progesterone, prostaglandins, and EGFlike factors in human ovulatory follicles. J Clin Endocrinol Metab. (2017) 102:1971-82. doi: 10.1210/jc.2016-3153

20. Hizaki H, Segi E, Sugimoto Y, Hirose M, Saji T, Ushikubi F., et al. Abortive expansion of the cumulus and impaired fertility in mice lacking the prostaglandin E receptor subtype EP(2). Proc Natl Acad Sci USA. (1999) 96:10501-6.

21. Luo B, Lin Y, Jiang S, Huang L, Yao H, Zhuang Q., et al. Endoplasmic reticulum stress eIF2alpha-ATF4 pathway-mediated cyclooxygenase-2 induction regulates cadmium-induced autophagy in kidney. Cell Death Dis. (2016) 7:e2251. doi: 10.1038/cddis.2016.78

22. Jin J, Zhao L, Zou W, Shen W, Zhang H, He Q. Activation of cyclooxygenase2 by ATF4 during endoplasmic reticulum stress regulates kidney podocyte autophagy induced by lupus nephritis. Cell Physiol Biochem. (2018) 48:753-64. doi: 10.1159/000491904

23. Cho HK, Cheong KJ, Kim HY, Cheong J. Endoplasmic reticulum stress induced by hepatitis $\mathrm{B}$ virus $\mathrm{X}$ protein enhances cyclo-oxygenase 2 expression via activating transcription factor 4. Biochem J. (2011) 435:431-9. doi: 10.1042/bj20102071

24. Rotterdam ESHRE/ASRM-Sponsored PCOS Consensus Workshop Group. Revised 2003 consensus on diagnostic criteria and long-term health risks related to polycystic ovary syndrome (PCOS). Hum Reprod. (2004) 19:41-7.

25. Matsubara H, Ikuta K, Ozaki Y, Suzuki Y, Suzuki N, Sato T., et al. Gonadotropins and cytokines affect luteal function through control of apoptosis in human luteinized granulosa cells. J Clin Endocrinol Metabol. (2000) 85:1620-6. doi: 10.1210/jcem.85.4.6509

26. Cheng JC, Fang L, Chang HM, Sun YP, Leung PC. hCG-induced Sprouty2 mediates amphiregulin-stimulated COX-2/PGE2 up-regulation in human granulosa cells: a potential mechanism for the OHSS. Sci Rep. (2016) 6:31675. doi: $10.1038 /$ srep31675

27. Popova E, Krivokharchenko A, Ganten D, Bader M. Comparison between PMSG- and FSH-induced superovulation for the generation of transgenic rats. Mol Reprod Dev. (2002) 63:177-82. doi: 10.1002/mrd. 10173

28. Sahin FK, Sahin SB, Balik G, et al. Does low pentraxin-3 levels associate with polycystic ovary syndrome and obesity? Int J Clin Exp Med. (2014) 7:3512-9. 
29. Duffy DM. Novel contraceptive targets to inhibit ovulation: the prostaglandin E2 pathway. Hum Reprod Update (2015) 21:652-70. doi: 10.1093/humupd/dmv026

30. Shimada M, Hernandez-Gonzalez I, Gonzalez-Robayna I, Richards JS. Paracrine and autocrine regulation of epidermal growth factor-like factors in cumulus oocyte complexes and granulosa cells: key roles for prostaglandin synthase 2 and progesterone receptor. Mol Endocrinol. (2006) 20:1352-65. doi: 10.1210/me.2005-0504

31. Matsuno Y, Onuma A, Fujioka YA, Yasuhara K, Fujii W, Naito K, et al. Effects of exosome-like vesicles on cumulus expansion in pigs in vitro. J Reprod Dev. (2017) 63:51-8. doi: 10.1262/jrd.2016-124

32. Curry TE Jr, Smith MF. Impact of extracellular matrix remodeling on ovulation and the folliculo-luteal transition. Semin Reprod Med. (2006) 24:228-41. doi: 10.1055/s-2006-948552

33. van Galen P, Kreso A, Mbong N, Kent DG, Fitzmaurice T, Chambers JE, et al. The unfolded protein response governs integrity of the haematopoietic stem-cell pool during stress. Nature (2014) 510:268-72. doi: 10.1038/nature 13228

34. Kuuranne T, Ahola L, Pussinen C, Leinonen A. Analysis of human chorionic gonadotropin (hCG): application of routine immunological methods for initial testing and confirmation analysis in doping control. Drug Test Anal. (2013) 5:614-8. doi: 10.1002/ dta. 1449
35. Liu J, Luo LF, Wang DL, Wang WX, Zhu JL, Li YC., et al. Cadmium induces ovarian granulosa cell damage by activating pERK-eIF2alpha-ATF4 through endoplasmic reticulum (ER) stressdagger. Biol Reprod. (2018). doi: 10.1093/biolre/ioy169. [Epub ahead of print].

36. Duffy DM, Stouffer RL. Follicular administration of a cyclooxygenase inhibitor can prevent oocyte release without alteration of normal luteal function in rhesus monkeys. Hum Reprod. (2002) 17:2825-31.

37. Hester KE, Harper MJ, Duffy DM. Oral administration of the cyclooxygenase2 (COX-2) inhibitor meloxicam blocks ovulation in non-human primates when administered to simulate emergency contraception. Hum Reprod. (2010) 25:360-7. doi: 10.1093/humrep/dep424

Conflict of Interest Statement: The authors declare that the research was conducted in the absence of any commercial or financial relationships that could be construed as a potential conflict of interest.

Copyright (c) $2018 \mathrm{Di}$, Liu, Li, Yao, Hong, Chen, Li and Du. This is an open-access article distributed under the terms of the Creative Commons Attribution License (CC $B Y)$. The use, distribution or reproduction in other forums is permitted, provided the original author(s) and the copyright owner(s) are credited and that the original publication in this journal is cited, in accordance with accepted academic practice. No use, distribution or reproduction is permitted which does not comply with these terms. 